\title{
Rectangle Transformation Problem *
}

\author{
Shaojiang Wang ${ }^{1,3,4}$, Kun $\mathrm{He}^{2,3,5}$, Yicheng Pan ${ }^{\dagger 1,4}$, Mingji Xia ${ }^{1,4}$ \\ 1. State Key Laboratory of Computer Science, \\ Institute of Software, Chinese Academy of Sciences \\ 2. CAS Key Lab of Network Data Science and Technology, \\ Institute of Computing Technology, Chinese Academy of Sciences \\ 3. University of Chinese Academy of Sciences \\ 4. Email: \{wangsj, yicheng, mingji\}@ios.ac.cn \\ 5. Email: hekun@ict.ac.cn
}

\begin{abstract}
In this paper, we propose the rectangle transformation problem (RTP) and its variants. RTP asks for a transformation by a rectangle partition between two rectangles of the same area. We are interested in the minimum RTP which requires to minimize the partition size. We mainly focus on the strict rectangle transformation problem (SRTP) in which rotation is not allowed in transforming. We show that SRTP has no finite solution if the ratio of the two parallel side lengths of input rectangles is irrational. So we turn to its complement denoted by SIRTP, in which case all side lengths can be assumed integral. We give a polynomial time algorithm ALGSIRTP which gives a solution at most $q / p+O(\sqrt{p})$ to $\operatorname{SIRTP}(p, q)(q \geq p)$, where $p$ and $q$ are two integer side lengths of input rectangles $p \times q$ and $q \times p$, and so ALGSIRTP is a $O(\sqrt{p})$-approximation algorithm for minimum $\operatorname{SIRTP}(p, q)$. On the other hand, we show that there is not constant solution to $\operatorname{SIRTP}(p, q)$ for all integers $p$ and $q(q>p)$ even though the ratio $q / p$ is within any constant range. We also raise a series of open questions for the research along this line.
\end{abstract}

*This work is supported in part by the 973 Program of China Grants No. 2014CB340302, and in part by the 973 Program of China Grants No. 2016 YFB1000201.

${ }^{\dagger}$ Corresponding author 


\section{Introduction}

We consider a practical problem in the Belt and Road initiative hosted by China. Freight amongst different countries and areas, by train, ship or plane etc, needs to transfer between transport facilities. To move cubic boxes between containers, suppose that each container is a cube, and the source container and target container usually have different specifications, i.e., different lengths, widths and heights. A problem is how to design a series of standard boxes to move between transport facilities of different specifications easily. For example, a practical challenge of building the Mongolia, China and Russia economic corridor infrastructure is their railway gauge differences, in which a key issue is the container between standard gauge and broad gauge conversion [7].

Suppose that two containers has the same volume, and we want to design a series of boxes such that they fully fills in each container perfectly. 11 When moving from one container to the other, the number of moving times is hopefully minimized, which means that the number of boxes is minimized.

A variant of this problem is a simplification by letting each box have the same height, or say in practice, no cover on the containers which, however, have the same floor area. So this problem reduces from 3-dimensional to 2-dimensional. Another variant is prohibiting rotation of each box, which in practice means that no rotation happens in moving boxes because of machinery constraints. In this paper, we propose the rectangle transformation problem and some of its variants to formulate the 2-dimensional version of this problem.

\subsection{Definitions and Problems}

We begin with defining rectangle partitions and isomorphic rectangle partitions as follows.

Definition 1.1. (Rectangle partitions) $A$ rectangle partition $\mathcal{P}$ on a rectangle $M$ is a partition on $M$ such that each module of $\mathcal{P}$ is a rectangle.

Definition 1.2. (Isomorphic rectangle partitions) Suppose that $M_{1}$ and $M_{2}$ are two rectangles of the same area. We say that two rectangle partitions $\mathcal{P}_{1}$ and $\mathcal{P}_{2}$ on $M_{1}$ and $M_{2}$, respectively, are isomorphic if $\mathcal{P}_{1}$ and $\mathcal{P}_{2}$ (two sets of modules)

\footnotetext{
${ }^{1}$ Usually, two containers are not exactly of the same volume. However, for some reasons such as compaction and safety in transportation, the boxes is supposed to be piled up in a cubic shape. This can be considered equivalent to transform between two same volumed containers.
} 
are exactly the same. That is, there is a one-one mapping between $\mathcal{P}_{1}$ and $\mathcal{P}_{2}$ such that each pair of modules (smaller rectangles from $\mathcal{P}_{1}$ and $\mathcal{P}_{2}$, respectively) related by this mapping have the same length and width.

In the above definition, rotation is allowed in identifying each pair of modules in the one-one mapping. If rotation is not needed, then we say that $\mathcal{P}_{1}$ and $\mathcal{P}_{2}$ are strictly isomorphic rectangle partitions. The rectangle transformation problem can be formulated as follows.

Problem 1.1. (Rectangle transformation problem, RTP for short) Let $M_{1}$ and $M_{2}$ be two rectangles $a \times b$ and $c \times d\left(a, b, c, d \in \mathbb{R}^{+}\right)$, respectively. Suppose that $a b=c d$, that is, $M_{1}$ and $M_{2}$ have the same area. The rectangle transformation problem requires to find a pair of isomorphic rectangle partitions $\mathcal{P}_{1}$ and $\mathcal{P}_{2}$ for $M_{1}$ and $M_{2}$, respectively.

The minimum RTP is the optimization problem such that the size (the number of modules) of $\mathcal{P}_{1}$ (or of $\mathcal{P}_{2}$, equivalently) is minimized. If $\mathcal{P}_{1}$ and $\mathcal{P}_{2}$ are required to be strictly isomorphic, then we call the RTP to be strict RTP (SRTP). Given input $a \times b$ and $c \times d$, the (strict) rectangle transformation problem is essentially requiring a transformation by a (strict) rectangle partition from $M_{1}$ to $M_{2}$.

Suppose that real numbers $a \geq c \geq d \geq b>0$ and $a b=c d$. ${ }^{2}$ We formulate the minimum RTP with input $a \times b$ and $c \times d$ as $\operatorname{RTP}(a, b, c, d)$. Similarly, ignoring the size relationship between $c$ and $d, \operatorname{SRTP}(a, b, c, d)$ can be defined if we clarify the parallel sides of the two rectangles, without loss of generality, $a$ and $c$.

An interesting observation for $\operatorname{SRTP}(a, b, c, d)$ is that, when we shrink a pair of parallel sides, for example, $a$ and $c$, for $d / a$ time, we get two rectangles of size $d \times b$ and $c d / a \times d$. Since $c d / a=b$, we in fact get two identical rectangles which are identified by $90^{\circ}$ rotation. A solution to this new pair of rectangles implies a solution to the original pair of rectangles by an $a / d$ time stretch on corresponding sides, and vice versa. Since SRTP prohibits rotation, the new problem $\operatorname{SRTP}(d, b, b, d)$ is not easy yet, but it is equivalent to $\operatorname{SRTP}(a, b, c, d)$. Thus, we can omit two parameters and define $\operatorname{SRTP}(p, q)$ for real numbers $p$ and $q$ to be the SRTP which requires strictly isomorphic rectangle partitions between rectangles $p \times q$ and $q \times p$.

The above is a general statement of (strict) RTP. In fact, for some cases, there might be no isomorphic rectangle partitions of finite size. For example, there are no finite strictly isomorphic rectangle partitions for $M_{1}=1 \times 2$ and $M_{2}=$ $\sqrt{2} \times \sqrt{2}$. Generally, we have the following theorem.

\footnotetext{
${ }^{2}$ We use " $\geq$ " rather than " $>$ " for generality although $a=c$ means a trivial case.
} 
Theorem 1.1. If $p / q$ is irrational, then there is no solution of finite size to $\operatorname{SRTP}(p, q)$.

We will prove this theorem in Section 4 . In the rest part of this paper, we focus on the case that $a, b, c, d \in \mathbb{Q}^{+}$, which turns to be equivalent to restricting $a, b, c, d \in \mathbb{Z}^{+}$for the reason of stretch technique, where $\mathbb{Z}^{+}$denotes the set of positive integers. In this case, we call RTP to be integral rectangle transformation problem (IRTP) and SRTP to be strict integral rectangle transformation problem (SIRTP). Both IRTP and SIRTP have obviously a trivial solution of size $a b$ (or equivalently $c d$ ). But to find an minimum solution is not easy yet. Similar to the definitions of $\operatorname{RTP}(a, b, c, d)$ and $\operatorname{SRTP}(p, q)$, we also define $\operatorname{IRTP}(a, b, c, d)$ and $\operatorname{SIRTP}(p, q)$. (Note that after transforming $\operatorname{SIRTP}(a, b, c, d)$ by the stretch technique, $\operatorname{SIRTP}(d, b, b, d)$ has an integer input also.) Because of the stretch technique, $\operatorname{SIRTP}(p, q)$ is equivalent to $\operatorname{SRTP}(p, q)$ when $p / q$ is rational, which is the complemental case of Theorem 1.1. Moreover, we can always assume that $a, b, c, d$ are mutually co-prime for $\operatorname{IRTP}(a, b, c, d)$ and $p, q$ are co-prime for $\operatorname{SIRTP}(p, q)$.

One thing we have to emphasize is that, for $\operatorname{IRTP}(a, b, c, d)$ or $\operatorname{SIRTP}(p, q)$, the description of a rectangle partition of size at most $a b$ or $p q$, respectively, might be of super-polynomial length of the input size. However, the representation of its size which is an integer at most $a b$ or $p q$ is of polynomial length. So from now on, we always assume the output of RTP (or IRTP, SRTP, SIRTP) to be the size of one of the isomorphic rectangle partitions.

\subsection{Relations to Other Partitioning Problems}

It has been known as Wallace-Bolyai-Gerwien theorem for centuries that a polygon can be dissected into any other polygon of the same area. Precisely, it states that two polygons are equidecomposable in terms of finitely many triangles if and only if they have the same area. However, for the dissections of a certain shape other than triangle, e.g. for our study, dissecting a rectangle into rectangles, the problem becomes quite different. Sometimes the equidecomposability is easy for rectangle partitions, but how to find an optimal partition is completely not known, and also rarely studied.

There are several optimization problems about geometrical dissections having been considered. The most famous one is triangulation, which requires a maximal partition of the convex hull of a set of points in a plane into triangles by using these points as triangle vertices. Many optimization criteria have been studied, for example, optimizing the minimum or maximum angle [9, 3] and the minimum 
weighted triangulation problem [5, 8]. The minimum-weight triangulation problem asks for a triangulation of a given point set that minimizes the sum of the edge lengths, and it has been proved to be NP-hard [8]. Generally, the deterministic version for the minimum number of pieces for polygon transformation, which is known as $k$-piece dissection problem, has also been proved to be NP-hard [2].

For rectangles, the problem of minimizing the largest perimeter of modules in rectangle partition of a certain size has been analyzed [4, 1]. Another interesting result states that if a rectangle is partitioned by rectangles each of which has at least an integer side, then the partitioned rectangle has at least an integer side [11].

However, as far as we know, no optimization problem on transformations of a certain geometrical figure by basic modules other than triangles has been studied. There are also few algorithmic studies for dissection problems. Our study opens this research for rectangles.

\subsection{Main Results and Techniques}

A straightforward method for a non-trivial solution to $\operatorname{IRTP}(a, b, c, d)(a \geq c \geq$ $d \geq b$ ) is using the Euclidean algorithm (that is, the successive division method) which proceeds by a greedy heuristic. At the beginning, align two adjacent sides of both rectangles at a corner arbitrarily, for example, align $a$ with $c$ and $b$ with d. 3 Then cut $a$ into $\lfloor a / c\rfloor$ segments and accordingly cut $d$ into $\lfloor d / b\rfloor$ segments (note that $a / c=d / b$ ). So $\lfloor a / c\rfloor$ many identical rectangles of size $c \times b$ in both rectangles are identified. The following task is to transform the unidentified parts $(a-\lfloor a / c\rfloor \cdot c) \times b$ and $c \times(d-\lfloor d / b\rfloor \cdot b)$ of the same area by isomorphic rectangle partitions, which is a subproblem of smaller scale. It proceeds recursively until getting a subproblem of transforming two identical rectangles. The procedure must end since the algorithm preserves integer side lengths in each subproblem and the least unit is $1 \times 1$ square in the integer case. The total number of modules is the sum of the integer parts of the ratios which can be calculated in $O\left(\log ^{3} a\right)$ time since the area of each subproblem halves in each round. So the Euclidean algorithm halts in polynomial time. The Euclidean algorithm works for $\operatorname{SIRTP}(p, q)$ also. The only difference is that, since rotation is not allowed, when align two adjacent sides of both rectangles, two vertical sides and two horizontal

\footnotetext{
${ }^{3}$ The other way to align adjacent sides is to align $a$ with $d$ and $b$ with $c$ (as shown in Figure 1). Any rule can be raised here to determined which way should be chosen. But the greedy strategy based on the fact that $\lfloor a / c\rfloor \leq\lfloor a / d\rfloor$ may not be always the best rule. For example, consider $\operatorname{IRTP}(15,2,6,5)$.
} 
sides are certainly aligned and successively divided, respectively, and so all the modules are squares.

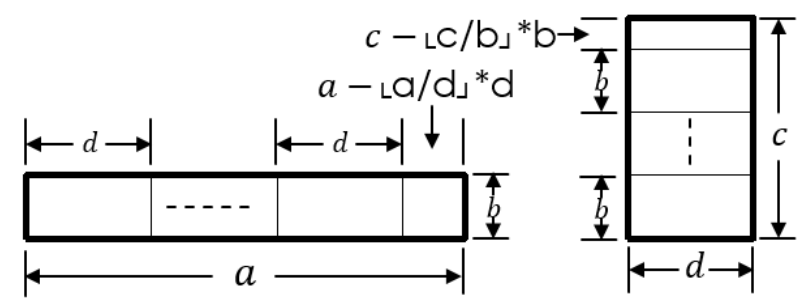

Figure 1: Illustration of one step of the Euclidean algorithm.

Note that a trivial lower bound for $\operatorname{IRTP}(a, b, c, d)(a \geq c \geq d \geq b)$ is $\lceil a / c\rceil$ since any rectangle with one side of length larger than $c$ cannot be contained in the one $c \times d$. For the same reason, $\lceil q / p\rceil$ is a trivial lower bound for $\operatorname{SIRTP}(p, q)$ if $q \geq p$. Thus, the Euclidean algorithm with the greedy strategy in the rule to determine the way of side alignments gives the optimal solution $a / c$ for $\operatorname{IRTP}(a, b, c, d)$ if $c \mid a$, and the optimal solution $q / p$ for $\operatorname{SIRTP}(p, q)$ if $p \mid q$. For the general case, we do not yet know whether the Euclidean algorithm (with a certain rule in determine the alignment of sides in each round) is a good algorithm for $\operatorname{IRTP}(a, b, c, d)$, but the following example indicates that it works badly for $\operatorname{SIRTP}(p, q)$. Consider $\operatorname{SIRTP}(p, p+1)$. In the first step, the Euclidean algorithm divides the rectangle of size $p \times(p+1)($ resp. $(p+1) \times p)$ into a square of size $p \times p$ and a slim rectangle of size $p \times 1$ (resp. $1 \times p$ ). But the only way in the subproblem of transforming $p \times 1$ to $1 \times p$ is cutting both of them into $p$ many $1 \times 1$ unit squares since $p$ is a trivial lower bound for this subproblem. Thus the algorithm gives the solution $p+1$ to $\operatorname{SIRTP}(p, p+1)$.

In this paper, we focus on SIRTP and establish the algorithm ALGSIRTP (shown in Section 2.2 which gives a solution at most $q / p+O(\sqrt{p})$ to $\operatorname{SIRTP}(p, q)$ $(q \geq p)$.

Theorem 1.2. Suppose that $p, q \in \mathbb{Z}^{+}$and $q \geq p$. ALGSIRTP is a polynomial time algorithm for $\operatorname{SIRTP}(p, q)$ and gives a solution at most $q / p+O(\sqrt{p})$.

Since $\lceil q / p\rceil$ is a lower bound, if $q=\Omega\left(p^{3 / 2}\right)$, then ALGSIRTP gives a solution to minimum $\operatorname{SIRTP}(p, q)$ with a constant approximation ratio. In general, it is a $O(\sqrt{p})$-approximation algorithm.

The main techniques used in ALGSIRTP are a square-transfer heuristic and its combination with Euclidean. The square-transfer technique means to build a 
square to transfer for two parts that are hard to transform directly. For instance, consider $\operatorname{SIRTP}(p, p+1)$. After the first step of the Euclidean algorithm, two slim rectangles $p \times 1$ and $1 \times p$ are left after cutting off a $p \times p$ square as a common module. Transforming these two rectangles directly is costly, but if they can fill in a common part in the $p \times p$ square simultaneously by partitioning into few modules, then they can be transferred in this part easily. For the case that $\sqrt{p}$ is an integer, rectangles $p \times 1$ and $1 \times p$ can be transferred by a $\sqrt{p} \times \sqrt{p}$ square. This heuristic is illustrated in Section 2.1. The algorithm in Theorem 1.2 is a recursive hybrid algorithm of Euclidean and square-transfer. It is given in Section 2.2.

Generally, the lower bound $\lceil q / p\rceil$ becomes small if $p$ and $q$ are close. An extreme case is minimum $\operatorname{SIRTP}(p, p+1)$ for which the Euclidean algorithm gives a $\Omega(p)$-approximation solution compared with the trivial lower bound $\lceil(p+$ $1) / p\rceil=2$. For more general lower bound, we have the following theorem.

Theorem 1.3. For any constants $\varepsilon>0$ and $m \in \mathbb{Z}^{+}$, there are positive integers $p$ and $q$ satisfying $p<q<(1+\varepsilon) p$ such that the minimum solution to $\operatorname{SIRTP}(p, q)$ is more than $m$.

Theorem 1.3 means that there is not constant solution independent of $p$ and $q$ to general $\operatorname{SIRTP}(p, q)$. The main technique used in its proof is to define the pattern of a rectangle partition. Two pairs of isomorphic rectangle partitions are equivalent if their two corresponding pairs of rectangle partitions have the same pattern under a fixed one-one map, respectively. Then we show a surprising lemma that all the equivalent pairs can only deal with $\operatorname{SIRTP}(p, q)$ for a fixed value of $p / q$. Constant size of a partition implies a constant number of patterns and also a constant number of pattern pairs, which can only deal with a constant number of values of $p / q$ in the range $p<q<(1+\varepsilon) p$ for any $\varepsilon>0$. Then Theorem 1.3 follows immediately. We prove it formally in Section 3 .

\section{The SIRTP Algorithm}

In this section, we give an algorithm which gives a solution at most $\lfloor q / p\rfloor+$ $8 \sqrt{p}+10 \log _{2} p$ to $\operatorname{SIRTP}(p, q)$ for $q \geq p$. At the beginning, we illustrate the square-transfer heuristic by the instance $\operatorname{SIRTP}(p, p+1)$. 


\subsection{The Square-Transfer Heuristic}

Given rectangles $p \times(p+1)$ and $(p+1) \times p$ for which $\sqrt{p}$ is an integer, we show that there is a pair of isomorphic partitions of size $2 \sqrt{p}+2$. First, we partition these two rectangles, respectively, into two modules, one of which is a $p \times p$ square. Then to transform for the left parts $p \times 1$ and $1 \times p$, we cut off a square $\sqrt{p} \times \sqrt{p}$ in the left bottom corner of each $p \times p$ square in both original rectangles. Then the rectangle $p \times(p+1)$, cut the slim rectangle $p \times 1$ into $\sqrt{p}$ segments, each of which is $\sqrt{p} \times 1$, and meanwhile, cut the $\sqrt{p} \times \sqrt{p}$ square into $\sqrt{p}$ many $1 \times \sqrt{p}$ rectangles (as illustrated in Figure 2(a) . Symmetrically, for the rectangle $(p+1) \times p$, cut the slim rectangle $1 \times p$ into $\sqrt{p}$ segments, each of which is $1 \times \sqrt{p}$, and meanwhile, cut the $\sqrt{p} \times \sqrt{p}$ square into $\sqrt{p}$ many $\sqrt{p} \times 1$ rectangles (as illustrated in Figure 2(b) $)$. Then cutting the rest part of the module $p \times p$ into two rectangles leads to a pair of isomorphic rectangle partitions of size $2 \sqrt{p}+2$. The key point for which this can be done is that the two slim rectangles can be cut off into few parts to fill in a common area (the $\sqrt{p} \times \sqrt{p}$ square) in a large module.

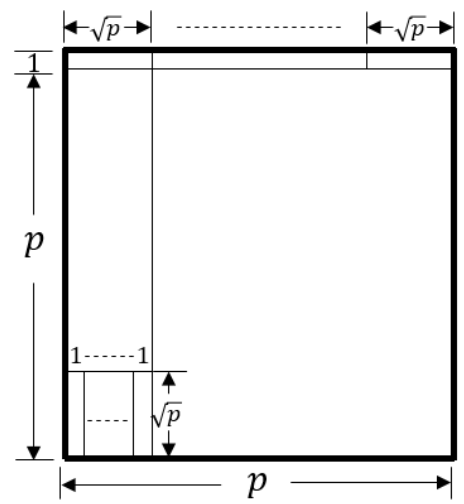

(a) Rectangle partition on $p \times(p+1)$

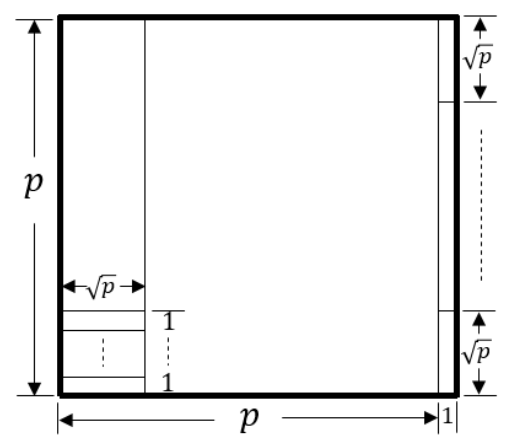

(b) Rectangle partition on $(p+1) \times p$

Figure 2: Illustration of the square-transfer heuristic for $\operatorname{SIRTP}(p, p+1)$ when $\sqrt{p}$ is an integer.

If $\sqrt{p}$ is not an integer, then we first cut each slim rectangles into $\lfloor\sqrt{p}\rfloor+1$ segments, in which each of the first $\lfloor\sqrt{p}\rfloor$ segments has length $\lfloor\sqrt{p}\rfloor$ and width 1. Then they can be transferred by a $\lfloor\sqrt{p}\rfloor \times\lfloor\sqrt{p}\rfloor$ square in the $p \times p$ square. The rest segment of each slim rectangles has length $p-\lfloor\sqrt{p}\rfloor^{2}$ and width 1 . Since $p-\lfloor\sqrt{p}\rfloor^{2} \leq p-(\sqrt{p}-1)^{2}=2 \sqrt{p}-1$, these two segments can be transformed directly by at most $2 \sqrt{p}-1$ unit squares, which leads to a pair of isomorphic rectangle partitions of size at most $4 \sqrt{p}+1$. 


\subsection{The Hybrid Algorithm of Euclidean and Square-Transfer}

We turn to the general case of $\operatorname{SIRTP}(p, q)$. The idea is to use the Euclidean step or the square-transfer step recursively for different cases of subproblems. Note that the idea of square-transfer can be easily generalized to $\operatorname{SIRTP}(p, p+\Delta)$ if $\Delta$ is far less than $p$. The method is to choose a proper length of segments for the less slim rectangles $p \times \Delta$ and $\Delta \times p$ such that all of them make up of a square. A key observation is that transforming the left parts of two rectangles is exactly a subproblem of much smaller area. This is also true for each Euclidean step in the Euclidean algorithm. So if $\Delta$ is close to $p$, we use the Euclidean step, and otherwise, we use the square-transfer step. This recursion process terminates within $2\left\lceil\log _{2} p\right\rceil+1$ steps if we guarantee the length of the shorter side in each subproblem halves in every two rounds.

Next, we state the algorithm firstly, and then show its correctness.

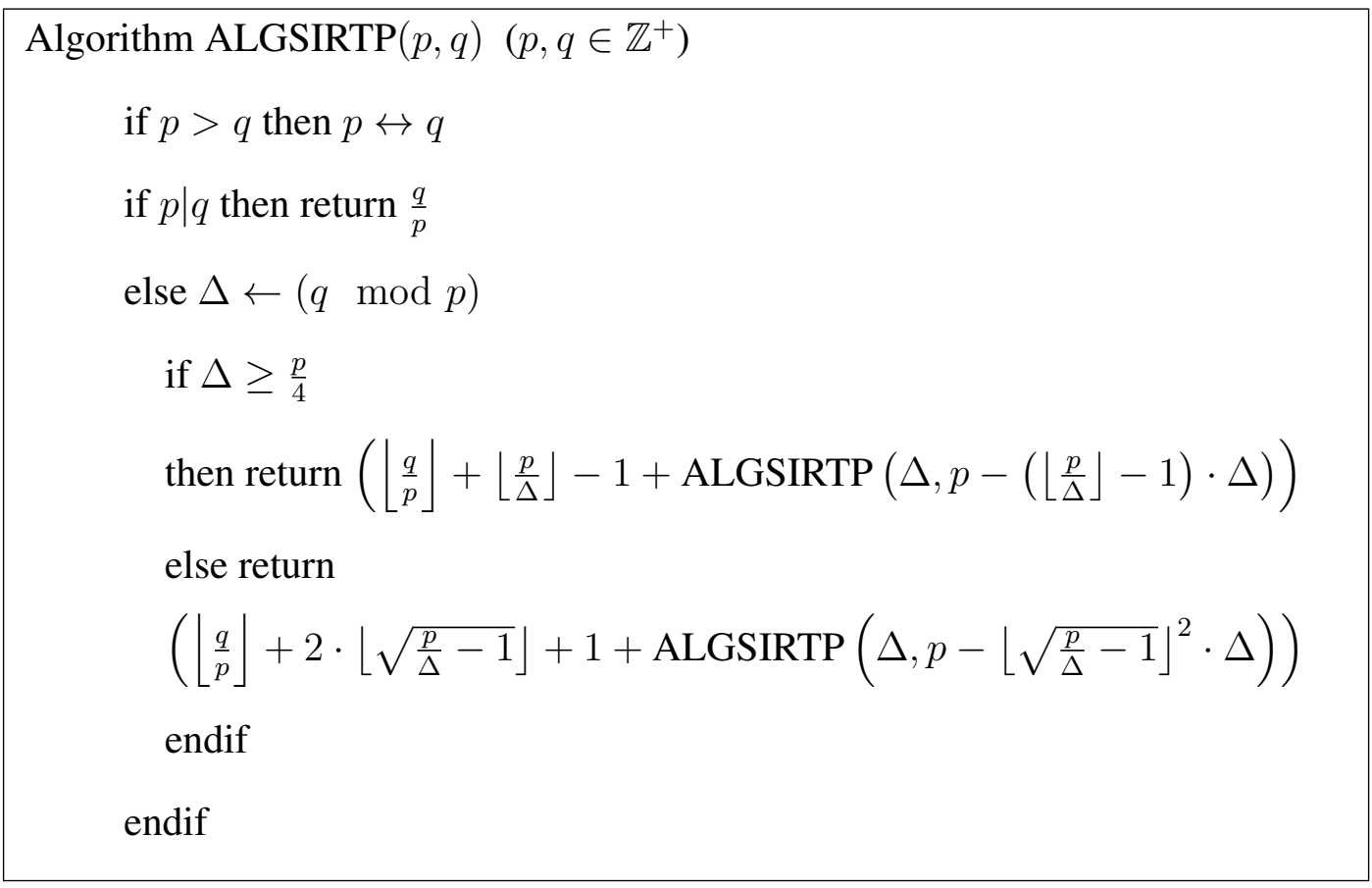

Note that in each round of recursions, ALGSIRTP calls itself as subroutine with smaller integer input. So it must terminate after finite steps. Then we prove Theorem 1.2.

Proof. First, we show that ALGSIRTP is a polynomial time algorithm. Note that the input length is $\left\lceil\log _{2} p\right\rceil+\left\lceil\log _{2} q\right\rceil$. Since division and mod can be calculated 
in log-square time, we only have to show that the number of recursions, denoted by $\ell$, is at most $O(\log p)$, which implies a time complexity $O\left(\log q \log p+\log ^{3} p\right)$.

We show that the length of the shorter side becomes no more than half in every successive recursions. In the step stated in $\operatorname{ALGSIRTP}(p, q)$, note that $\Delta>0$,

$$
p-\left(\left\lfloor\frac{p}{\Delta}\right\rfloor-1\right) \cdot \Delta \geq p-\left(\frac{p}{\Delta}-1\right) \cdot \Delta=\Delta
$$

and

$$
p-\left\lfloor\sqrt{\frac{p}{\Delta}-1}\right\rfloor^{2} \cdot \Delta \geq p-\left(\frac{p}{\Delta}-1\right) \cdot \Delta=\Delta .
$$

The length of the shorter side in each subroutine is $\Delta$. If $\Delta \leq p / 2$, then the length of the shorter side in each subroutine halves. Otherwise, $\Delta>p / 2$ in this round, and thus the first subroutine $\operatorname{ALGSIRTP}(\Delta, p)$ will be executed in the next round. Now the value $\Delta^{\prime}:=(p \bmod \Delta)=p-\Delta<p / 2$. So in the next round, the length of the shorter side becomes $\Delta^{\prime}$, which is at most half of $p$. Therefore, $\ell \leq 2\left\lceil\log _{2} p\right\rceil+1$.

Next, we show the correctness of the algorithm. Let $p_{i}, q_{i}$ and $\Delta_{i}(0 \leq i \leq \ell)$ be the parameters in the $i$-th recursion, where $p_{i} \leq q_{i}, \Delta_{i}=\left(q_{i} \bmod p_{i}\right), p_{0}=p$ and $q_{0}=q$. We will show that for each $0 \leq i<\ell$, the problem $\operatorname{SIRTP}\left(p_{i}, q_{i}\right)$ can be reduced to $\operatorname{SIRTP}\left(p_{i+1}, q_{i+1}\right)$ by adding $\left\lfloor q_{i} / p_{i}\right\rfloor+\left\lfloor p_{i} / \Delta_{i}\right\rfloor-1$ many rectangles if $\Delta_{i} \geq p_{i} / 4$, and $\left\lfloor q_{i} / p_{i}\right\rfloor+2 \cdot\left\lfloor\sqrt{p_{i} / \Delta_{i}-1}\right\rfloor+1$ many rectangles, otherwise.

For each $0 \leq i<\ell$, in the $i$-th round, we cut off $\left\lfloor q_{i} / p_{i}\right\rfloor$ many rectangles from both rectangles $p_{i} \times q_{i}$ and $q_{i} \times p_{i}$ just as what we do in a Euclidean step. When $\Delta_{i} \geq p_{i} / 4$, we cut off $\left\lfloor p_{i} / \Delta_{i}\right\rfloor-1$ more squares $\Delta_{i} \times \Delta_{i}$ from both rectangles $p_{i} \times \Delta_{i}$ and $\Delta_{i} \times p_{i}$ which are left in the two rectangles $p_{i} \times q_{i}$ and $q_{i} \times p_{i}$, respectively. Then this induces the subproblem $\operatorname{SIRTP}\left(p_{i+1}, q_{i+1}\right)$ where $p_{i+1}=\Delta_{i}$ and $q_{i+1}=p_{i}-\left(\left\lfloor p_{i} / \Delta_{i}\right\rfloor-1\right) \cdot \Delta_{i}$, while $\left\lfloor q_{i} / p_{i}\right\rfloor+\left\lfloor p_{i} / \Delta_{i}\right\rfloor-1$ many rectangles are added. This way of partition is illustrated in Figure 3 , where $\left\lfloor q_{i} / p_{i}\right\rfloor$ is simplified to be 1 and the shaded areas shows the $\operatorname{subproblem} \operatorname{SIRTP}\left(p_{i+1}, q_{i+1}\right)$.

When $\Delta_{i}<p_{i} / 4$, we use the square-transfer heuristic. We cut off $\left\lfloor\sqrt{p_{i} / \Delta_{i}-1}\right\rfloor$ more identical rectangles $s_{i} \times \Delta_{i}$ (resp. $\Delta_{i} \times s_{i}$ ) from $p_{i} \times \Delta_{i}$ (resp. $\Delta_{i} \times p_{i}$ ), where $s_{i}=\left\lfloor\sqrt{p_{i} / \Delta_{i}-1}\right\rfloor \cdot \Delta_{i}$. These rectangles make up of the square $s_{i} \times s_{i}$, which can be cut off as a transfer square from one of the larger squares $p_{i} \times p_{i}$ (there must be one). Thus this induces the subproblem $\operatorname{SIRTP}\left(p_{i+1}, q_{i+1}\right)$ where $p_{i+1}=\Delta_{i}$ and $q_{i+1}=p_{i}-\left\lfloor\sqrt{p_{i} / \Delta_{i}-1}\right\rfloor^{2} \cdot \Delta_{i}$, while $\left\lfloor q_{i} / p_{i}\right\rfloor+2 \cdot\left\lfloor\sqrt{p_{i} / \Delta_{i}-1}\right\rfloor+1$ many rectangles are added. This is illustrated in Figure 4, where $\left\lfloor q_{i} / p_{i}\right\rfloor$ is also simplified to be 1 and the shaded areas shows the subproblem $\operatorname{SIRTP}\left(p_{i+1}, q_{i+1}\right)$. 


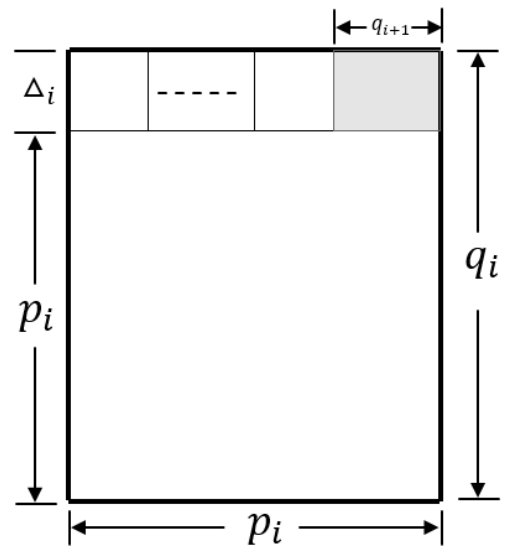

(a) Rectangle partition on $p_{i} \times q_{i}$

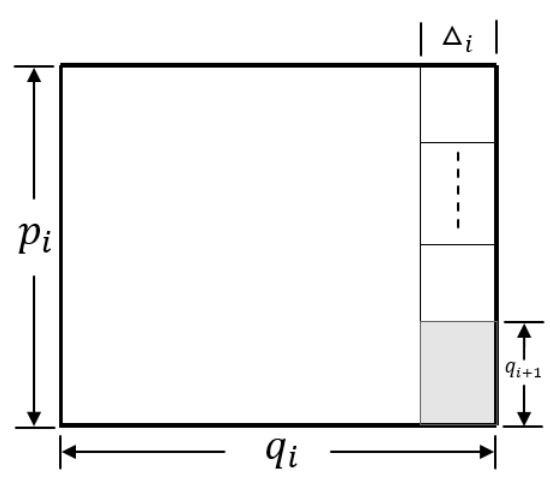

(b) Rectangle partition on $q_{i} \times p_{i}$

Figure 3: Illustration of one step of recursion for $\operatorname{SIRTP}\left(p_{i}, q_{i}\right)$ when $\Delta_{i} \geq p_{i} / 4$.

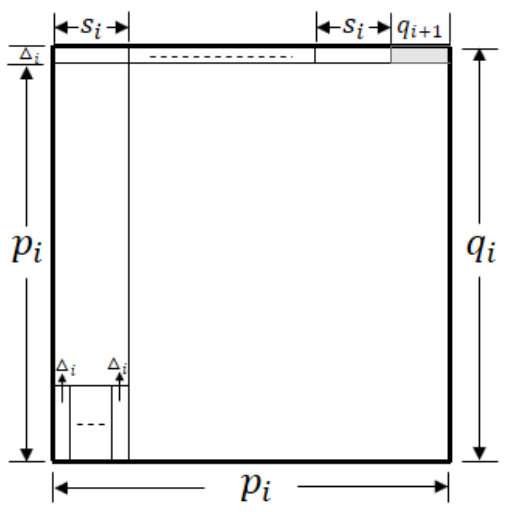

(a) Rectangle partition on $p_{i} \times q_{i}$

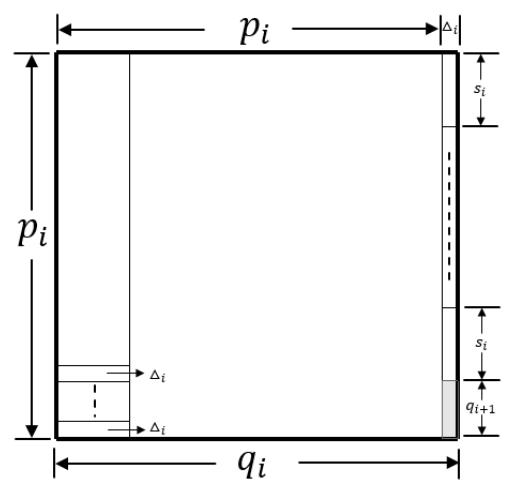

(b) Rectangle partition on $q_{i} \times p_{i}$

Figure 4: Illustration of one step of recursion for $\operatorname{SIRTP}\left(p_{i}, q_{i}\right)$ when $\Delta_{i}<p_{i} / 4$. 
Then we only have to prove the upper bound in Theorem 1.2. Concretely, we show that the output of $\operatorname{ALGSIRTP}(p, q)$ is at most $\lfloor q / p\rfloor+8 \sqrt{p}+10 \log _{2} p$ for $q \geq p$. To this end, we prove the following lemma.

Lemma 2.1. For each $0 \leq i \leq \ell, \operatorname{ALGSIRTP}\left(p_{i}, q_{i}\right) \leq\left\lfloor q_{i} / p_{i}\right\rfloor+8 \sqrt{p_{i}}+\log _{2} p_{i}+$ $4(\ell-i)$ for $q_{i} \geq p_{i}$.

Proof. We prove this lemma by induction on $i$ from $\ell$ down to 0 . For the basis case that $p_{\ell} \mid q_{\ell}, \operatorname{ALGSIRTP}\left(p_{\ell}, q_{\ell}\right)=q_{\ell} / p_{\ell}$. The lemma holds for $i=\ell$. For the inductive step, assume that the lemma holds for $i=k+1$. Then for $i=k$, we consider the two cases that $\Delta_{k} \geq p_{k} / 4$ and $\Delta_{k}<p_{k} / 4$, respectively.

By the way of partitions, if $\Delta_{k} \geq p_{k} / 4$, then $q_{k+1}=p_{k}-\left(\left\lfloor p_{k} / \Delta_{k}\right\rfloor-1\right) \cdot \Delta_{k}$ and $p_{k+1}=\Delta_{k}<p_{k}$. We have

$$
\begin{aligned}
& \operatorname{ALGSIRTP}\left(p_{k}, q_{k}\right) \\
\leq & \left\lfloor\frac{q_{k}}{p_{k}}\right\rfloor+\left\lfloor\frac{p_{k}}{\Delta_{k}}\right\rfloor-1+\operatorname{ALGSIRTP}\left(p_{k+1}, q_{k+1}\right) \\
\leq & \left\lfloor\frac{q_{k}}{p_{k}}\right\rfloor+\left\lfloor\frac{p_{k}}{\Delta_{k}}\right\rfloor-1+\left\lfloor\frac{q_{k+1}}{p_{k+1}}\right\rfloor+8 \sqrt{p_{k+1}}+\log _{2} p_{k+1}+4[\ell-(k+1)] \\
\leq & \left\lfloor\frac{q_{k}}{p_{k}}\right\rfloor+\left\lfloor\frac{p_{k}}{\Delta_{k}}\right\rfloor-1+\frac{p_{k}}{\Delta_{k}}-\left(\left\lfloor\frac{p_{k}}{\Delta_{k}}\right\rfloor-1\right)+8 \sqrt{p_{k+1}}+\log _{2} p_{k+1}+4(\ell-k)-4 \\
< & \left\lfloor\frac{q_{k}}{p_{k}}\right\rfloor+8 \sqrt{p_{k}}+\log _{2} p_{k}+4(\ell-k)+\frac{p_{k}}{\Delta_{k}}-4 \\
\leq & \left\lfloor\frac{q_{k}}{p_{k}}\right\rfloor+8 \sqrt{p_{k}}+\log _{2} p_{k}+4(\ell-k) .
\end{aligned}
$$

The lemma holds for $i=k$.

If $\Delta_{k}<p_{k} / 4$, then $q_{k+1}=p_{k}-\left\lfloor\sqrt{p_{k} / \Delta_{k}-1}\right\rfloor^{2} \cdot \Delta_{k}$ and $p_{k+1}=\Delta_{k}$. We 
have

$$
\begin{aligned}
& \operatorname{ALGSIRTP}\left(p_{k}, q_{k}\right) \\
\leq & \left\lfloor\frac{q_{k}}{p_{k}}\right\rfloor+2 \cdot\left\lfloor\sqrt{\frac{p_{k}}{\Delta_{k}}-1}\right\rfloor+1+\operatorname{ALGSIRTP}\left(p_{k+1}, q_{k+1}\right) \\
\leq & \left\lfloor\frac{q_{k}}{p_{k}}\right\rfloor+2 \cdot\left\lfloor\sqrt{\frac{p_{k}}{\Delta_{k}}-1}\right\rfloor+1+\left\lfloor\frac{q_{k+1}}{p_{k+1}}\right\rfloor+8 \sqrt{p_{k+1}}+\log _{2} p_{k+1}+4[\ell-(k+1)] \\
\leq & \left\lfloor\frac{q_{k}}{p_{k}}\right\rfloor+2 \cdot\left\lfloor\sqrt{\frac{p_{k}}{\Delta_{k}}-1}\right\rfloor+1+\frac{p_{k}}{\Delta_{k}}-\left\lfloor\left.\sqrt{\frac{p_{k}}{\Delta_{k}}-1}\right|^{2}+8 \sqrt{p_{k+1}}+\log _{2} p_{k+1}\right. \\
& +4[\ell-(k+1)] \\
\leq & \left\lfloor\frac{q_{k}}{p_{k}}\right\rfloor+2 \cdot \sqrt{\frac{p_{k}}{\Delta_{k}}-1}+1+\frac{p_{k}}{\Delta_{k}}-\left(\sqrt{\frac{p_{k}}{\Delta_{k}}-1}-1\right)^{2}+8 \sqrt{\Delta_{k}}+\log _{2} \Delta_{k} \\
& +4[\ell-(k+1)] \\
< & \left\lfloor\frac{q_{k}}{p_{k}}\right\rfloor+4 \cdot \sqrt{\frac{p_{k}}{\Delta_{k}}-1}+1+8 \sqrt{\frac{p_{k}}{4}}+\log _{2} \frac{p_{k}}{4}+4(\ell-k)-4 \\
< & \left\lfloor\frac{q_{k}}{p_{k}}\right\rfloor+8 \sqrt{p_{k}}+\log _{2} p_{k}+4(\ell-k) .
\end{aligned}
$$

The lemma holds for $i=k$.

Combining these two cases, Lemma 2.1 follows.

Since $\ell \leq 2\left\lceil\log _{2} p\right\rceil+1$, the upper bound is obtained when $i=0$ in Lemma 2.1. Theorem 1.2 has been proved.

By the proof of Theorem 1.2, ALGSIRTP gives not only a solution to $\operatorname{SIRTP}(p, q)$, but also a partition method on the two rectangles by the choice in each round of recursions, and the coordinates of partition lines are integers.

Recall that in $\operatorname{Section} 1.1$, we reduce $\operatorname{SIRTP}(a, b, c, d)(a \geq c \geq d \geq b)$ to $\operatorname{SIRTP}(d, b, b, d)$. Another reduction is to $\operatorname{SIRTP}(a, c, c, a)$ by multiplying $c / b$ to the side of lengths $b$ and $d$. Since $a / d=c / b$, ALGSIRTP gives the same solution to $\operatorname{SIRTP}(d, b, b, d)$ and $\operatorname{SIRTP}(a, c, c, a)$ at most $d / b+O(\sqrt{b})$, and thus the same solution to $\operatorname{SIRTP}(a, b, c, d)$. The latter one does not necessarily implies partitions with integer coordinate lines.

For the general case for $\operatorname{SRTP}(p, q)$ that $p / q$ is rational, because of the stretch technique, we can assume that $p$ and $q$ are two rational numbers, denoted by $p=$ $p_{1} / p_{2}$ and $q=q_{1} / q_{2}$ the irreducible fractions, where $p_{i}, q_{i} \in \mathbb{Z}^{+}$for $i \in\{1,2\}$. 
We can also convert it to the integeral case by multiplying the least common multiple $\operatorname{lcm}\left(p_{2}, q_{2}\right)$ to them. Then assuming $q \geq p$, ALGSIRTP gives a solution at most $q / p+O\left(\sqrt{p_{1} q_{2} / g}\right)$, where $g=\operatorname{gcd}\left(p_{2}, q_{2}\right)$ is the greatest common divisor of $p_{2}$ and $q_{2}$. This is not a $O(\sqrt{p})$-approximation solution to minimum $\operatorname{SRTP}(p, q)$ and ALGSIRTP is not be a poly $(\log p)$ time algorithm any more. However, in this case, if we are given $p_{1}, q_{1}, p_{2}, q_{2}$ as input, then ALGSIRTP halts in poly $\left(\log p_{2} q_{1}\right)$ time.

\section{No Constant Lower Bound}

In this section, we prove Theorem 1.3 . First, we define the pattern of a rectangle partition and the equivalence of pairs of isomorphic rectangle partitions. Second, we show that if two equivalent pairs of isomorphic rectangle partitions solve $\operatorname{SIRTP}(p, q)$ and $\operatorname{SIRTP}\left(p^{\prime}, q^{\prime}\right)$, respectively, then $p / q=p^{\prime} / q^{\prime}$. This means that an equivalent class of isomorphic rectangle partition pairs can only deal with the $\operatorname{SIRTP}(p, q)$ for a fixed value of $p / q$. So a constant number of equivalent classes cannot deal with too many $\operatorname{SIRTP}(p, q)$ instances.

\subsection{The Pattern of A Rectangle Partition}

Suppose that $\mathcal{P}$ is a rectangle partition of size $k$ on rectangle $M$. An observation is that, except the four corners of $M$, every the intersection among partition lines and $M$ 's sides is of shape " $\perp$ " or cross. By extending all the partition lines to be face-to-face, all the " $\perp$ "-shaped intersections become crosses, while some new cross intersections might emerge. Such extension makes a grid partition on $M$ and we call it the extension of $\mathcal{P}$. Suppose that in this extension, there are $r$ rectangles in each row and $c$ rectangles in each column.

For each integer $1 \leq i \leq k$, define matrix $M_{i}$ to be a 0 - 1 matrix of size $r \times c$, in which the $(u \times v)$-th entry $(1 \leq u \leq r, 1 \leq v \leq c)$ is 1 if and only if the $(u \times v)$-th rectangle in the extension belongs to the $i$-th module of $\mathcal{P}$. We define the pattern of $\mathcal{P}$ to be the $k$-tuple $\left(M_{1}, M_{2}, \ldots, M_{k}\right)$. We say that two rectangle partitions of size $k$ have the same pattern if they have exactly the same $k$-tuple, including the size and the entries of each $M_{i}$. Figure 5 illustrates the pattern of a rectangle partition of size 6 , in which each $M_{i}$ for $1 \leq i \leq 6$ is a $0-1$ matrix of size $3 \times 4$, and for example, the second row of $M_{3}$ is $\left(\begin{array}{llll}1 & 1 & 1 & 0\end{array}\right)$ and other two rows are $\left(\begin{array}{llll}0 & 0 & 0 & 0\end{array}\right)$. 


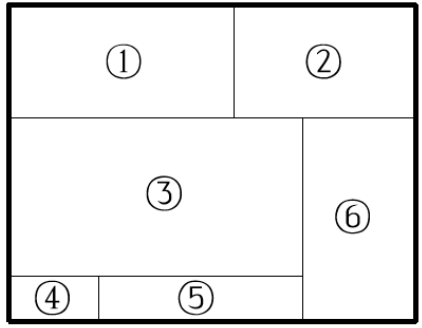

(a) Original rectangle partition

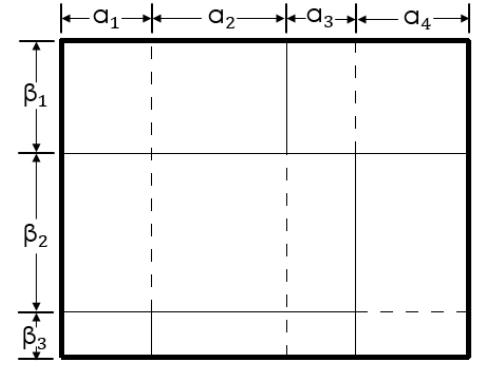

(b) Extension of the original rectangle partition

Figure 5: Illustration of the pattern of a rectangle partition.

Note that for each $i$ the rank of $M_{i}$ is exactly one since each module of $\mathcal{P}$ is a rectangle. Denote the non-zero row of $M_{i}$ by $\vec{r}_{i}$ and the non-zero column of $M_{i}$ by $\vec{c}_{i}$. Let $l_{i} \times h_{i}$ be the $i$-th module of $\mathcal{P}$ and $\alpha_{u} \times \beta_{v}$ be the $(u \times v)$-th rectangle in the extension. Let $\vec{\alpha}_{i}=\left(\alpha_{1}, \alpha_{2}, \ldots, \alpha_{c}\right)$ and $\vec{\beta}_{i}=\left(\beta_{1}, \beta_{2}, \ldots, \beta_{r}\right)$ be the two length vectors for the two adjacent sides, respectively. Thus for each $1 \leq i \leq k$,

$$
l_{i}=\left\langle\vec{\alpha}, \vec{r}_{i}\right\rangle
$$

and

$$
h_{i}=\left\langle\vec{\beta}, \vec{c}_{i}\right\rangle
$$

\subsection{Proof of Theorem 1.3}

Then we prove Theorem 1.3 .

Proof. For two pairs of isomorphic rectangle partitions $\left(\mathcal{P}_{1}, \mathcal{P}_{2}\right)$ and $\left(\mathcal{P}_{1}^{\prime}, \mathcal{P}_{2}^{\prime}\right)$, suppose that all four partitions have size $k$. Because of isomorphism, there is a one-one map, denoted by $\pi$ (resp. $\left.\pi^{\prime}\right):[k] \rightarrow[k]$, to identify the modules in $\left(\mathcal{P}_{1}\right.$ and $\left.\mathcal{P}_{2}\right)$ (resp. in $\left(\mathcal{P}_{1}^{\prime}\right.$ and $\left.\mathcal{P}_{2}^{\prime}\right)$ ). We say that $\left(\mathcal{P}_{1}, \mathcal{P}_{2}\right)$ and $\left(\mathcal{P}_{1}^{\prime}, \mathcal{P}_{2}^{\prime}\right)$ are equivalent, if $\pi=\pi^{\prime}, \mathcal{P}_{1}, \mathcal{P}_{1}^{\prime}$ have the same pattern and $\mathcal{P}_{2}, \mathcal{P}_{2}^{\prime}$ have the same pattern also. For simplification, we can assume that $\pi$ and $\pi^{\prime}$ are both the identity map, and each pair of $M_{i}$ 's are equal accordingly.

Lemma 3.1. Suppose that $\left(\mathcal{P}_{1}, \mathcal{P}_{2}\right)$ and $\left(\mathcal{P}_{1}^{\prime}, \mathcal{P}_{2}^{\prime}\right)$ are equivalent isomorphic rectangle partition pairs for $\operatorname{SIRTP}(p, q)$ and $\operatorname{SIRTP}\left(p^{\prime}, q^{\prime}\right)$, respectively $(q \geq p$, $\left.q^{\prime} \geq p^{\prime}\right)$. Then $p / q=p^{\prime} / q^{\prime}$. 
Proof. For $\mathcal{P}_{1}$, let $l_{i} \times h_{i}$ be the $i$-th module. In its extension, let $\vec{\alpha}^{(p)}$ be the length vector and $r_{i}^{(1)}$ be the non-zero row of $M_{i}$ parallel to the horizontal side $p$. Similarly, for $\mathcal{P}_{1}^{\prime}$, we define $l_{i}^{\prime} \times h_{i}^{\prime}, \vec{\alpha}^{\left(p^{\prime}\right)}, r_{i}^{(1)}$ (note that $\mathcal{P}_{1}$ and $\mathcal{P}_{1}^{\prime}$ have the same pattern, and so they have the same $r_{i}^{(1)}$ ); for $\mathcal{P}_{2}$, we define $l_{i} \times h_{i}$ (note that $\mathcal{P}_{1}$ and $\mathcal{P}_{2}$ are isomorphic, and so the modules are the same), $\vec{\alpha}^{(q)}, r_{i}^{(2)}$; for $\mathcal{P}_{2}^{\prime}$, we define $l_{i}^{\prime} \times h_{i}^{\prime}, \vec{\alpha}^{\left(q^{\prime}\right)}, r_{i}^{(2)}$. Then by the definition of pattern, for each $i \in[k]$,

$$
l_{i}^{\prime}=\left\langle\vec{\alpha}^{\left(p^{\prime}\right)}, r_{i}^{(1)}\right\rangle=\left\langle\vec{\alpha}^{\left(q^{\prime}\right)}, r_{i}^{(2)}\right\rangle,
$$

Since

$$
p \cdot q=\sum_{i=1}^{k} l_{i} \cdot h_{i}=\sum_{i=1}^{k}\left\langle\vec{\alpha}^{(p)}, r_{i}^{(1)}\right\rangle \cdot h_{i},
$$

replacing each $\alpha_{i}^{(p)}$ by $\alpha_{i}^{\left(p^{\prime}\right)}$ while keeping each $h_{i}$ makes a rectangle partition of the same pattern on the new rectangle $p^{\prime} \times q$. That is,

$$
p^{\prime} \cdot q=\sum_{i=1}^{k}\left\langle\vec{\alpha}^{\left(p^{\prime}\right)}, r_{i}^{(1)}\right\rangle \cdot h_{i}=\sum_{i=1}^{k} l_{i}^{\prime} \cdot h_{i} .
$$

On the other hand, for the same reason, since

$$
q \cdot p=\sum_{i=1}^{k} l_{i} \cdot h_{i}=\sum_{i=1}^{k}\left\langle\vec{\alpha}^{(q)}, r_{i}^{(2)}\right\rangle \cdot h_{i}
$$

replacing each $\alpha_{i}^{(q)}$ by $\alpha_{i}^{\left(q^{\prime}\right)}$ while keeping each $h_{i}$ makes a rectangle partition of the same pattern on the new rectangle $q^{\prime} \times p$. That is,

$$
q^{\prime} \cdot p=\sum_{i=1}^{k}\left\langle\vec{\alpha}^{\left(q^{\prime}\right)}, r_{i}^{(2)}\right\rangle \cdot h_{i}=\sum_{i=1}^{k} l_{i}^{\prime} \cdot h_{i}
$$

Comparing Equations (1) with (2), we have

$$
p^{\prime} \cdot q=\sum_{i=1}^{k} l_{i}^{\prime} \cdot h_{i}=q^{\prime} \cdot p .
$$

Lemma 3.1 follows. 
By Lemma 3.1, we know that equivalent isomorphic rectangle partition pairs can only deal with $\operatorname{SIRTP}(p, q)$ for a fixed ratio $p / q$. Note that for a rectangle partition $\mathcal{P}$ of size $k$, there are at most $k-1$ many face-to-face extensions of partition lines in each direction. So the number of rectangles in its extension is at most $k^{2}$. When $k$ is a constant, the number of pattern of $\mathcal{P}$ is also a constant. Thus, the number, denoted by $f(k)$, of equivalent pairs of isomorphic rectangle partitions of size $k$ is also a constant. However, for any constants $\varepsilon>0$ and $m \in \mathbb{Z}^{+}$, there are more than $f(m)$ many values of $p / q$, which means more than $f(m)$ many instances of $\operatorname{SIRTP}(p, q)$. By Lemma 3.1, so many instances cannot be all solved by all pairs of isomorphic rectangle partitions of size $m$. This completes the proof of Theorem 1.3 .

\section{Proof of Theorem 1.1}

We define a sort of rectangle partition named slat rectangle partition that will be used in this proof.

Definition 4.1. (Slat rectangle partition) A slat rectangle partition $\mathcal{P}$ is a rectangle partition on a rectangle $M$, such that if two horizontal sides of two modules overlap, then they are identical.

In other words, when going along any vertical partition line, you will reach the horizontal sides of $M$, without interruption by any horizontal side of any module except the horizontal sides of $M$. The slat rectangle partition, looks like wooden floor composed of many slats.

Lemma 4.1. If $\operatorname{SRTP}(p, q)$ has a solution then there is a solution such that one partition in the solution is a slat rectangle partition.

Proof. Suppose $\operatorname{SRTP}(p, q)$ has a solution $\left(\mathcal{P}_{1}, \mathcal{P}_{2}\right)$. For each rectangle in $\mathcal{P}_{1}$, we extend its vertical sides. Since some modules of $\mathcal{P}_{1}$ are cut into rectangles by the extended lines, we get a refinement of $\mathcal{P}_{1}$, denoted by $\mathcal{P}_{1}^{\prime}$. Obviously, $\mathcal{P}_{1}^{\prime}$ is a slat rectangle partition.

We apply the same refinement to $\mathcal{P}_{2}$ and get $\mathcal{P}_{2}^{\prime}$. Obviously, $\left(\mathcal{P}_{1}^{\prime}, \mathcal{P}_{2}^{\prime}\right)$ is also a solution.

\footnotetext{
${ }^{4}$ In this section, we resume the original meaning of a solution to SRTP, that is, a pair of isomorphic rectangle partitions for two rectangles, rather than the partition size.
} 
We define the width multi-set of rectangle partition, to be the multi-set of the widths of its rectangles, and define the width set to be the set of all widths.

Lemma 4.2. If $\operatorname{SRTP}(p, q)$ has a solution $\left(\mathcal{P}_{1}, \mathcal{P}_{2}\right)$, then there is a solution $\left(\mathcal{P}_{1}^{\prime}, \mathcal{P}_{2}^{\prime}\right)$ such that the width set is linear independent over rational numbers. Moreover, for any $i \in\{1,2\}$, if $\mathcal{P}_{i}$ is a slat rectangle partition, then $\mathcal{P}_{i}^{\prime}$ is a slat rectangle partition.

Proof. The general intuition of the proof is simple. Whenever there is a width in the width set that is a linear combination other widths, we cut all the rectangles of this width vertically into smaller rectangles according to the combination. While in the proof, we have to handle the negative coefficients in the linear combinations very carefully, since all widths are positive, and we can only cut it into positive segments.

Suppose that the width set of $\mathcal{P}_{1}$ and $\mathcal{P}_{2}$ is $\left\{x_{1}, x_{2}, \ldots, x_{n}\right\}$. We can cut all rectangles in $\mathcal{P}_{1}$ and $\mathcal{P}_{2}$ of width $x_{i}$ into $c$ rectangles of width $x_{1} / c$. Obviously, this operation, named mincing operation, changes a slat partition into another one, and keeps the isomorphism between two partitions.

Suppose that the rank of $\left\{x_{1}, x_{2}, \ldots, x_{n}\right\}$ is $r$. The proof is an induction on $n$. Whenever $n>r$, we construct a new solution, whose width set has size $n-1$ and rank $r$.

Because $n>r$, the width set is linearly dependent. Notice all widths are positive numbers. There must be a linear equation of the form $b_{1} y_{1}+b_{2} y_{2}+\cdots+$ $b_{s} y_{s}=c_{1} z_{1}+c_{2} z_{2}+\cdots+c_{t} z_{t}$, where the coefficients are positive integers, and $y_{1}, y_{2}, \ldots, y_{s}, z_{1}, z_{2}, \ldots, z_{t}$ are distinct elements from the width set, and $s, t$ are two positive integers satisfying $s+t \geq 2$.

We keep $y_{1}$ unchanged and mince each of other widths $y_{2}, \ldots, y_{s}, z_{1}, z_{2}, \ldots, z_{t}$ into $b_{1}$ pieces. Because $b_{1} y_{1}+b_{2} b_{1}\left(\frac{1}{b_{1}} y_{2}\right)+\cdots+b_{s} b_{1}\left(\frac{1}{b_{1}} y_{s}\right)=c_{1} b_{1}\left(\frac{1}{b_{1}} z_{1}\right)+$ $c_{2} b_{1}\left(\frac{1}{b_{1}} z_{2}\right)+\cdots+c_{t} b_{1}\left(\frac{1}{b_{1}} z_{t}\right)$, the new width set satisfies $y_{1}+b_{2} y_{2}^{\prime}+\cdots+b_{s} y_{s}^{\prime}=$ $c_{1} z_{1}^{\prime}+c_{2} z_{2}^{\prime}+\cdots+c_{t} z_{t}^{\prime}$. The new width set has size $s$ and still rank $r$, since it is linearly equivalent to the original set.

The purpose of the next step is to mince $y_{2}^{\prime}, \ldots, y_{s}^{\prime}$ tiny enough such that they can be put into the bins on the right side of the equation. There are $c_{1}$ bins of size $z_{1}^{\prime}, c_{2}$ bins of size $z_{2}^{\prime}$ and so on. There are $b_{2}$ commodities of size $y_{2}^{\prime}, b_{3}$ commodities of size $y_{2}^{\prime}$ and so on. The total room of these bins $c_{1} z_{1}^{\prime}+c_{2} z_{2}^{\prime}+\cdots+c_{t} z_{t}^{\prime}$ is $y_{1}$ larger than total size of the commodities $b_{2} y_{2}^{\prime}+\cdots+b_{s} y_{s}^{\prime}$. The requirement is that each bin in the same size category, leaves the same room unoccupied.

Let $N$ denote $c_{1}+c_{2}+\cdots+c_{t}$. We always mince the commodities into pieces of sizes no more than $\frac{y_{1}}{N}$. If we have fully utilized a bin such that no piece can be 
put into it, then the waste room is no more than $\frac{y_{1}}{N}$. In this way, we never waste too much, and the total room of left bins and the room of unsealed bins, is always larger the size of unpacked commodities.

We mince $y_{2}^{\prime}$ into $T c_{1}$ pieces, where $T$ is larger enough such that each piece has size no more than $\frac{y_{1}}{N}$. We put these pieces into the $c_{1}$ bins of size $z_{1}^{\prime}$ fairly. There are two cases. If all pieces are put into the $c_{1}$ bins, we go on to mince $y_{3}^{\prime}$ and put its pieces into bins in the same way, starting from these unsealed $c_{1}$ bins.

If there are pieces left, then each bin of size $z_{1}^{\prime}$ has a room of size $z_{2}^{\prime \prime}$ unoccupied. We seal these $c_{1}$ bins, the unoccupied room of each bin is no more than $\frac{y_{1}}{N}$, and it is unoccupied forever. We go on to put these pieces into the $c_{2}$ bins of size $z_{2}^{\prime}$. Now, there is a problem, if the number of left pieces is not a multiple of $c_{2}$, then we are not able to be fair to all the $c_{2}$ bins, and not sure to fulfill the requirement. The trick is simply mincing each pieces into $c_{2}$ subpieces, no matter the packed ones or the unpacked ones. The current size of each subpieces is $\frac{y_{2}^{\prime}}{T c_{1} c_{2}}$

Again, there are two cases, either we pack all subpieces of $y_{2}^{\prime}$, or we seal these $c_{2}$ bins and begin to pack them into the $c_{3}$ bins of size $z_{3}^{\prime}$. In the second case, we will mince each subpieces into $c_{3}$ subsubpieces.

We repeat this process, until all commodities are packed up. Suppose each $y_{i}^{\prime}$ is finally minced into pieces of size $y_{i}^{\prime \prime}, i=2,3, \ldots, s$. Suppose the left room of each bin of size $z_{i}^{\prime}$ is $z_{i}^{\prime \prime}, i=1,2, \ldots, t$. We show that $\left\{y_{2}^{\prime \prime}, \ldots, y_{s}^{\prime \prime}, z_{1}^{\prime \prime}, \ldots, z_{t}^{\prime \prime}\right\}$ can linearly express $\left\{y_{1}, y_{2}^{\prime}, \ldots, y_{s}^{\prime}, z_{1}^{\prime}, \ldots, z_{t}^{\prime}\right\}$. Each $y_{i}^{\prime}$ is a multiple of $y_{i}^{\prime \prime}$. Each $z_{i}^{\prime}$ is a linear combination of $z_{i}^{\prime \prime}$ and the sizes of pieces in the corresponding bin. And $y_{1}$ is a linear combination of $z_{i}^{\prime \prime}$, since it is the size of the total unoccupied room. Together the rest elements in the width set, we get a smaller new width set.

The new set has the same rank. We repeat this process, until the size of the width set becomes $r$.

Then we turn to prove Theorem 1.1 .

Proof. Assume that there is a finite solution to $\operatorname{SRTP}(p, q)$. By Lemma 4.1 and 4.2. there is a solution $\left(\mathcal{P}_{1}, \mathcal{P}_{2}\right)$, such that $\mathcal{P}_{1}$ is a slat rectangle partition and the width set $\left\{x_{1}, x_{2}, \ldots, x_{n}\right\}$ is linearly independent over rational numbers.

Consider the partition $\mathcal{P}_{1}$. The total height of all width $x_{1}$ modules is $s p$, where $s$ is an integer. Then consider the partition $\mathcal{P}_{2}$. Suppose that a horizontal line goes face-to-face. It also goes though some modules, and it is cut into segments by these modules. The length of each segments is equal to the width of the rectangle being cut. The total length $p$ is an integer coefficient linear combination $t_{1} x_{1}+$ 
$\cdots t_{n} x_{n}$ of $\left\{x_{1}, x_{2}, \ldots, x_{n}\right\}$. Because the width set is linearly independent over rational numbers, the combination realizing $p$ is unique.

When we move this horizontal line, we always get the same combination. If we scan the whole rectangle, whose height is $q$, from top to bottom, we find out that the total height of all width $x_{1}$ modules rectangles in partition $\mathcal{P}_{2}$ is $t_{1} q$. Since $\mathcal{P}_{1}$ is isomorphic to $\mathcal{P}_{2}, s p=t_{1} q$, which contradicts to the fact that $p / q$ is irrational. Theorem 1.1 has been proved.

\section{Conclusions and Future Discussions}

In this paper, we proposed the rectangle transformation problem (RTP), and defined its strict version (SRTP), integral version (IRTP) and their combination (SIRTP). We showed that $\operatorname{SRTP}(p, q)$ has no finite solution if the ratio of two side lengths $p / q$ is irrational. So we focused on the complemental case for $\operatorname{SRTP}(p, q)$ that $p$ and $q$ are integers. We gave an algorithm for $\operatorname{SRTP}(p, q)$ which gave a solution at most $q / p+O(\sqrt{p})$ (assume $q \geq p$ ), and showed that there is not any constant solution $k$ independent of $p$ and $q$ for $\operatorname{SRTP}(p, q)$ even if the ratio $q / p$ is in any constant range.

As a new problem, RTP and its variants leave a lot of open questions. We list some representative ones for the research along this line and discuss possible approaches to some of them.

(1) For $\operatorname{SIRTP}(p, q)$, is there a polynomial time algorithm that gives a better solution than ALGSIRTP, for example, $q / p+p^{\varepsilon}$ for some $\varepsilon<1 / 2$ or even $q / p+O(\log p)$ ? We conjecture that it is possible since the recursive step in ALGSIRTP seems to have a large development space. By the proof of Theorem 1.2, keeping the total number of recursions within $O(\log p)$, in each recursive step, additional $p^{\varepsilon}$ many rectangles will lead to an improvement to $q / p+p^{\varepsilon}$ and additional constant many rectangles will lead to an improvement to $q / p+O(\log p)$.

(2) The lower bound for $\operatorname{SIRTP}(p, q)$ seems to be able to be dependent on $p$ and $q$. A straight method is to improve the proof of Theorem 1.3 by finding an explicit relationship between the rectangle partition size and the number of patterns. Another question is whether this idea can be used to IRTP. It is clear that the pattern for IRTP can be defined similarly, but adjustments to side lengths on a pattern become more complicated. 
(3) Whether there is a good algorithm for minimum IRTP $(p, q)$ ? Here, "good" means a polynomial time algorithm that have a good approximation, or even gives the optimal solution. It is a possible approach to give a rule for the choice of the Euclidean algorithm in each recursive step as we discussed in Section 1.3 .

(4) For the negative direction of Question (3), what is the hardness of the deterministic version of minimum $\operatorname{IRTP}(p, q)$ and minimum $\operatorname{SIRTP}(p, q)$, that is, whether there is a solution at most $k$ ? If we restrict the partition lines to be of integer coordinate, a straight method to find the optimal solution is to enumerate the patterns for all pairs of isomorphic rectangle partitions along the integer coordinates. Even with this restriction, the running time of this method is just upper bounded by $\exp \{O(p, q)\}$. Guessing the patterns and the one-one map for the isomorphic rectangle partitions implies that the deterministic versions of minimum IRTP and minimum SIRTP with integral solution restriction are just both in NEXP. Without this restriction, we do not even know how to find the optimal solution regardless of running time. If Question (3) have a positive answer for the optimal solution, it will be amazing and quite interesting. Note that even though one of minimum IRTP and minimum SIRTP is easy, it cannot be implied that the other one is also easy. These two problems are very different.

(5) A more practical version of RTP is to relax the target rectangle to be of area $(1+\delta) S$, where $S$ is the area of the source rectangle. Then minimum RTP requires to minimize the module number in the rectangle partition of the source one such that all modules can be covered by the target one with all sides parallel to a boundary. This is a mixed scenario about partitioning and 2-dimensional bin packing. The latter and its generalization to 3dimensional case have been studied widely for a long time. The techniques raised there might be helpful. See [6, 10] for a survey.

(6) RTP and all of its variants can be generalized to 3-dimensional version. The two lower bounds in this paper is also true for 3-dimensional case, but the algorithm ALGSIRTP cannot be generalized directly. It is worthwhile to have a systematic study. 


\section{References}

[1] N. Alon and D. Kleitman. Partitioning a rectangle into small perimeter rectangles. Discrete Mathematics, 103, 111-119, 1992.

[2] J. Bosboom, E. D. Demaine. M. L. Demaine, J. Lynch, P.Manurangsi. M. Rudoy and A. Yodpinyanee. Dissection with the fewest pieces is hard, even to approximate. Japanese Conference on Discrete and Computational Geometry and Graphs, 37-48, 2015.

[3] H. Edelsbrunner, T. S. Tan and R. Waupotitsch. A polynomial time algorithm for the minmax angle triangulation. Proc. 6th ACM Symposium on Computational Geometry, 44-52, 1990.

[4] S. Kasif and R. Klette. A data allocation problem for SIMD systems. Center for Automation Research, TR 1292, 1983.

[5] E. L. Lloyd. On triangulations of a set of points in the plane. Proc. 18th IEEE Symposium on Foundations of Computer Science, 228-240, 1977.

[6] A. Lodi, S. Martello and D. Vigo. Recent advances on two-dimensional bin packing problems. Discrete Applied Mathematics, 123, 379-396, 2002.

[7] G. Luft. It takes a road - China's one belt one road initiative: an American response to the new silk road. Institute for the Analysis of Global Security, 2016.

[8] W. Mulzer and G. Rote. Minimum weight triangulation is NP-hard. Proc. 22th ACM Symposium on Computational Geometry, 1-10, 2006.

[9] D. M. Mount and A. Saalfeld. Globally-equiangular triangulations of cocircular points in $O(n \log n)$ time. Proc. 4th ACM Symposium on Computational Geometry, 143-152, 1988.

[10] V. T. Paschos. Paradigms of combinatorial optimization: problems and new approaches, Volume 2. John Wiley \& Sons, 107-129, 2013.

[11] S. Wagon. Fourteen proofs of a result about tiling a rectangle. The American Mathematical Monthly, 94, 601-617, 1987. 